\title{
Microwave Diversity Imaging using Six-port Reflectometer
}

\author{
Hsin-Chia Lu and Tah-Hsiung Chu* \\ Department of Electrical Engineering, \\ National Taiwan University, Taipei, Taiwan \\ Fax: 886-02-3638247, E-mail:leonardo@ew.ee.ntu.edu.tw, thc@ew.ee.ntu.edu.tw
}

\section{Introduction}

As a perfectly conducting convex object is illuminated with a monochromatic plane wave, under physical optics approximation, its range-normalized backscattered far field and the object characteristic function forms a Fourier transformation pair. This relationship is known as the Bojarski's identity $[1,2]$. To acquire the scattering information about the object more effectively, the frequency and angular diversity techniques are used [3]. Conventionally, both the amplitude and phase data of the object scattered field are measured by a vector network analyzer (VNA). However, VNA may have two limitations. One is the upper frequency limit, the other is its cost. Six-port reflectometer $[4,5]$ is known as an alternate approach to measure the reflection coefficient of microwave devices. In a six-port reflectometer, signals at its four output ports are linear combinations of the signals at the two input ports. After proper calibration, one can calculate the complex ratio of the two input signals from the power measurements at the four output ports. Therefore, by using the six-port reflectometer one can eliminate the coherent receiver in VNA, since only power measurements are involved.

In this paper, we use six-port reflectometer for microwave diversity imaging measurement in a compact range arrangement and develop the associated calibration method. In the calibration, a reference scattering object is placed at the object positioner with the use of a calibration circuit. Microwave diversity imaging experiments are conducted for three types of scattering objects, a metallic cylinder, four distributed line scatterers and a scaled B-52 aircraft model. The operation frequency is from $8 \mathrm{GHz}$ to $12 \mathrm{GHz}$.

\section{Experiment Arrangement and Calibration Procedure \\ A. Anechoic chamber and compact range arrangement}

As shown in Fig.1, the experiment arrangement has an anechoic chamber with a compact range facility to produce a plane wave illumination to the region of object positioner. The transmitting and receiving antennas are placed together with spacing about $15 \mathrm{~cm}$ as a backscattering arrangement. The main reflector and subreflector has the size about $2 \mathrm{~m}$ and 0.9 m in diameter respectively. Four sets of corrugated horns are operated in the frequency bands $8 \sim 12 \mathrm{GHz}, 12 \sim 18 \mathrm{GHz}, 18 \sim 26 \mathrm{GHz}$ and $26 \sim 40 \mathrm{GHz}$. In our measurement, the operation frequency is from $8 \mathrm{GHz}$ to $12 \mathrm{GHz}$.

In this arrangement, an HP 83620 synthesized sweeper is used as the signal source. The transmitted signal is then through a traveling wave tube amplifier (TWTA) to the transmitting corrugated horn. A calibration circuit is connected to the coupled port of a $10 \mathrm{~dB}$ coupler to provide the reference signal to the six-port circuit. The received object scattered field from the receiving corrugated horn then goes to the other input port of the six-port circuit. Amplitude values at the four output ports of six-port circuit are then recorded. The synthesized sweeper, object positioner and data acquisition instruments are linked to a Sun Sparc-10 workstation through IEEE 488 interface.

$0-7803-4178-/ 3 / 97 / \$ 10.00 \oplus 1997$ IEEE 


\section{B. Six-port reflectometer with calibration circuit}

The six-port reflectometer shown in Fig.l contains a six-port circuit and a calibration circuit. The six-port circuit consists of four $90^{\circ}$ hybrids. One input port of the six-port circuit samples the incident field through a $10 \mathrm{~dB}$ coupler, and the other input port receives the object scattered field.

The calibration circuit consists of an HP $8494 \mathrm{H}$ electronically controlled variable attenuator and a SEMPLEX MFR 60637 phase shifter. The attenuator has an attenuation range from 0 to $11 \mathrm{~dB}$ with $1 \mathrm{~dB}$ per step. The phase shifter is a coaxial line stretcher with about $80^{\circ}$ phase shift at $8 \mathrm{GHz}$. In the calibration measurement, a metallic cylinder with diameter of $25.4 \mathrm{~cm}$ is placed on the object positioner, with the cylinder front edge at the center of positioner. A total of fifteen sets of data are recorded by changing three attenuator positions and five phase shifter positions. The five parameters for characterizing the six-port reflectometer to an equivalent four-port reflectometer transformation are firstly solved. Note the attenuation and phase shift values are not required to be known. In solving the calibration parameters, singular value decomposition method is used. We then take one attenuator setting to be $0 \mathrm{~dB}$, one associated phase shifter setting to be $0^{\circ}$, and the measured reflection coefficient to be unity as the reference signal for the four-port reflectometer calibration. In this second step calibration, three parameters are solved from ten sets of previously recorded data, corresponding to two attenuation settings and five phase shifter settings. After the calibration, the calibration circuit is then set to the original settings for the microwave diversity imaging measurements.

After the described two calibration steps, the six-port measurement system becomes equivalent to a four-port (or VNA) measurement. One can then use the error model for anechoic chamber as $S_{m}=S_{c}+S_{t} S_{o} S_{r}$, where $S_{c}$ is the clutter response of the anechoic chamber, $S_{t}$ and $S_{r}$ are the responses for transmitting and receiving path including antenna and compact range. $S_{m}$ and $S_{o}$ are the measured and calibrated object response. Note in a microwave diversity imaging system, $S_{c}, S_{t}$ and $S_{r}$ are frequency responses only, and $S_{m}$ and $S_{o}$ are responses of frequency and view angle.

\section{Measurement procedure}

The calibration procedure of the developed microwave diversity imaging system using sixport reflectometer is summarized as the following.

1. Place a cylinder as the reference object with its edge at the center of positioner.

2. Use calibration circuit to calibrate the six-port reflectometer.

a. Set attenuator at $0 \mathrm{~dB}$ and phase shifter at 0 degree, then measure the six-port circuit output power values.

b. Change attenuator at three positions and phase shifter at five positions, then measure fifteen sets of the six-port circuit output power values.

c. Use the measured fifteen sets of power values to calibrate the six-port reflectometer. With the calibration parameters, measured power values from six-port circuit then give the complex reflection coefficient. In other word, the six-port reflectometer can be viewed as a conventional four-port reflectometer after this calibration procedure.

3. Use the reflection coefficients calculated from the calibration set to calibrate the four-port reflectometer as the step two calibration. The resulting response of the reference object is given as $S_{r e f}(f)$. 
4. Locate the scattering object on the positioner, measure the six-port output power values at the selected frequencies and viewing angles, then convert the measured object responses $S_{m}(f, \theta)$ in a polar format.

5. Measure the room clutter response $S_{c}(f)$, and the corrected object response is given as $S_{o}(f, \theta)=\frac{S_{m}(f, \theta)-S_{c}(f)}{S_{r e f}(f)-S_{c}(f)}$.

6. After acquiring the polar format of the scattering object response, a two-dimensional interpolation algorithm is used to transfer data from polar grids into rectangular grids for two-dimensional fast Fourier transform for image reconstruction.

\section{Experiment Results}

Experiment results of three different types of scattering objects, including a metallic cylinder, four distributed line scatterers and a B-52 72:1 scaled aircraft model, are shown in Fig. 3. The cylinder diameter is $25.4 \mathrm{~cm}$ and is placed in the center of object positioner. The four line scatterers are placed at $(12,0),(0,-8),(-3,5,6)$ and $(-10,-5)$ in $\mathrm{cm}$.

In data recording for cylinder, the range of viewing angle is from $0^{\circ}$ to $360^{\circ}$ for a total of 60 views, and the frequency used is stepped from $8 \mathrm{GHz}$ to $12 \mathrm{GHz}$ for 101 points. The range of viewing angle for line scatterers and B-52 scaled aircraft model is from $0^{\circ}$ to $90^{\circ}$ for 50 views and the same frequency range and steps as those used for cylinder. The reconstructed image of cylinder shows the shape of cylinder with correct dimension, whereas the reconstructed image of four line scatterers gives the correct positions of four line scatterers. The reconstructed image of B-52 72:1 scaled aircraft model shows clearly the images of fuselage, wings, engines and oil tanks.

\section{Conclusion}

Conventionally, the object scattered field for microwave diversity imaging is measured by using vector network analyzers. In this paper, we described the experiment arrangement and calibration procedure using a six-port reflectometer developed in our compact range facility. The reconstructed images show the same quality as those using vector network analyzer. These results indicate that with the use of six-port reflectometer one can not only reduce the cost of microwave diversity image facility, but also extend the operation frequency to the millimeter wave range.

\section{References}

[1] R. M. Lewis, "Physical optics inverse diffraction," IEEE Trans. Antennas Propagat., vol. AP-24, pp. 308-314, May 1969.

[2] N. N. Bojarski, "A survey of physical optics inverse scattering identity," IEEE Trans. Antennas Propagat., vol. AP-30, pp. 980-989, Sept. 1982.

[3] N. H. Farhat, "Microwave diversity imaging and automated target identification based on models of neural networks," Proc. IEEE., vol. 77, pp. 670-680, May 1989.

[4] C. A. Hoer, "The six-port coupler: a new approach to measuring voltage, current, power, impedance, and phase," IEEE Trans. Instrum. Meas., vol. IM-21, pp. 466-470, Nov. 1972.

[5] G. F. Engen and C. A. Hoer, "Application of an arbitrary 6-port junction to powermeasurement problem," IEEE Trans. Instrum. Meas., vol. IM-21, pp. 470-474, Nov. 1972. 


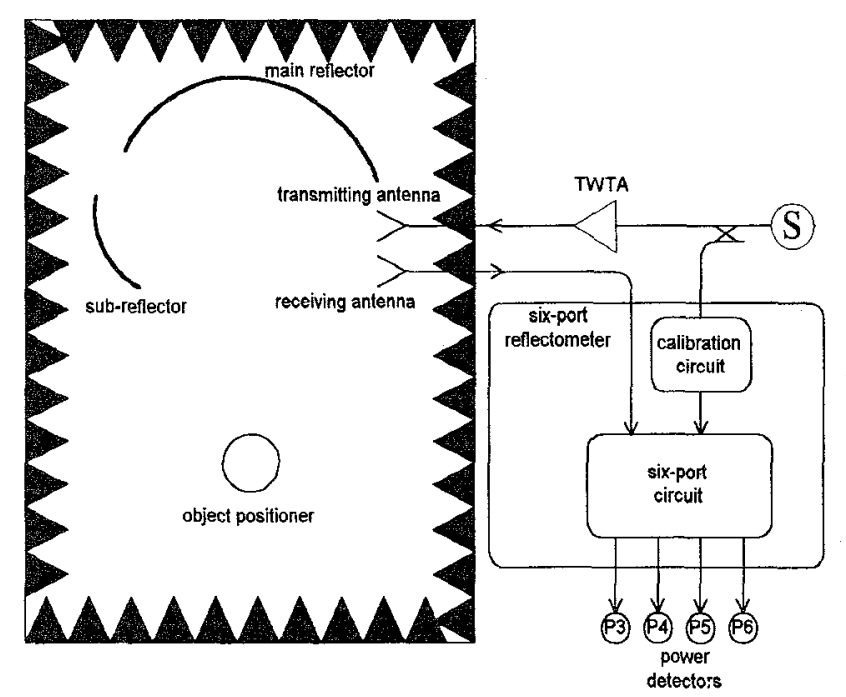

Figure 1 Experiment arrangement of microwave diversity imaging system using six-port reflectometer.
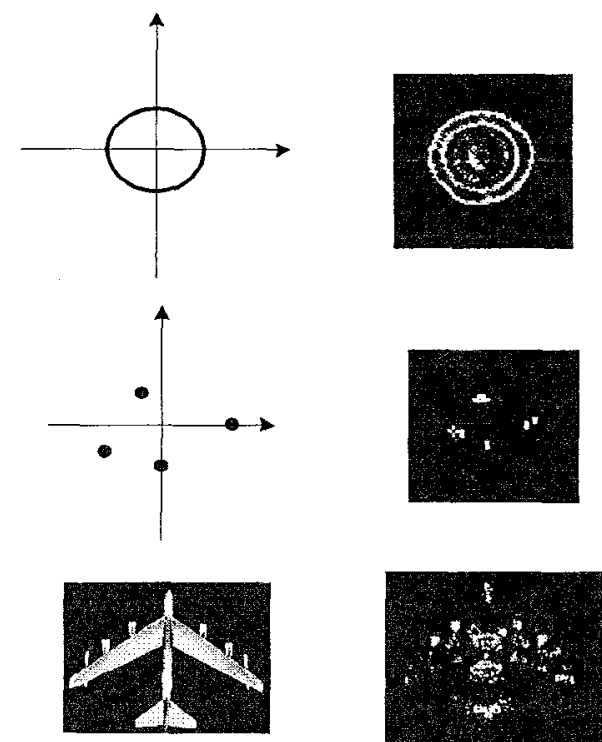

Figure 2 Experimental results of three different shapes of scattering objects including a metallic cylinder, four distributed line scatterers, and a B-52 72:1 scaled aircraft model. 